\title{
Computer Administering of the Psychological Investigations: Set-Relational Representation
}

\author{
Krasimir Yordzhev ${ }^{1}$, Ivelina Peneva ${ }^{2}$ \\ ${ }^{1}$ Faculty of Mathematics and Natural Sciences, South-West University, Blagoevgrad, Bulgaria \\ ${ }^{2}$ Department of Psychology, Faculty of Philosophy, South-West University, Blagoevgrad, Bulgaria \\ Email: \{yordzhev, ivelina_peneva\}@swu.bg
}

Received March 23, 2012; revised April 25, 2012; accepted May 5, 2012

\begin{abstract}
Computer administering of a psychological investigation is the computer representation of the entire procedure of psychological assessments - test construction, test implementation, results evaluation, storage and maintenance of the developed database, its statistical processing, analysis and interpretation. A mathematical description of psychological assessment with the aid of personality tests is discussed in this article. The set theory and the relational algebra are used in this description. A relational model of data, needed to design a computer system for automation of certain psychological assessments is given. Some finite sets and relation on them, which are necessary for creating a personality psychological test, are described. The described model could be used to develop real software for computer administering of any psychological test and there is full automation of the whole process: test construction, test implementation, result evaluation, storage of the developed database, statistical implementation, analysis and interpretation. A software project for computer administering personality psychological tests is suggested.
\end{abstract}

Keywords: Computer Administering; Computer Testing; Computer Psycho Diagnostic; Mathematical Modeling; Set Theory; Relational Algebra; Databases

\section{Introduction}

Psychological assessment will be called any psychologycal testing made with the help of a preliminary prepared test - a list of questions or statements, that the assessed person or group of people has to answer or to give their opinion for. The separate parts of the test are called items.

We will call computerized psychological assessment any psychological testing, where one or several phases of the testing are made with the use of a computer.

Computer administering of psychological tests is the computer representation of the entire procedure of psychological assessments - test construction, test implementation, results evaluation, storage and maintenance of the developed database, its statistical processing, analysis and interpretation.

Personality questionnaires are those psychological tests, which are purposed for description and evaluation of the characteristics of cognitive (behavioral), emotional and motivation sphere, the interpersonal relations and attitudes of an individual [1]. It's typical for the Personality questionnaires (in contrast to Achievement tests or Intelligence tests) that the items are questions or statements, for which answers the respondent has to report certain information concerning himself, his experience and relations. The format of the answers to items is also specific - most often they are described with the help of a finite set of preliminary known answers or statements, which we will mark by $A n s$. In our examinations we will accentuate mainly on finite sets of possible responds to each item of the test. For example Ans = " "Yes", "No" $\}, A n s=\{" T r u e ", " F a l s e "\}$, Ans = "I like it", "I don't like it"\}, Ans = \{"Often", "Sometimes", "Never"\}, Ans = \{"True", "I don't know", "False"\}, Ans = \{"Agree", "I'm not sure", "Disagree" $\}$. Items with rating scales are also used in practice, but we will examine just rating scales that represent definite and consequently discrete set of real numbers.

For more details about basic terms in personality psychological testing see for example in $[2,3]$.

There are different ways to structure, process and store data in a software product. Data and data links are abstraction of facts and relations from the real world [4]. Very often this abstraction is rather complex and requires a special mathematical model for its description - a data model. The use of one or other model means that in certain information system are chosen different principles for data structuring or data operation. The most widespread data model nowadays is the relational model, suggested for the first time by E. F. Codd at the begin- 
ning of the 1970s of the last century and described in a set of articles, one of the earliest is [5]. For this article E. F. Codd won a prestigious A. Turing award of the American Association for Computing Machinery in 1980.

Let the family of sets $D=\left\{D_{1}, D_{2}, \cdots, D_{m}\right\}$, which we will call domains, be given. Let's examine the Cartesian product:

$$
\begin{aligned}
W & =D_{i_{1}} \times D_{i_{2}} \times \cdots \times D_{i_{n}} \\
& =\left\{\left\langle x_{1}, x_{2}, \cdots, x_{n}\right\rangle \mid x_{k} \in D_{i_{k}}, D_{i_{k}} \in D, k=1,2, \cdots, n\right\}
\end{aligned}
$$

(It is possible for some $s$ and $t, D_{i_{s}}=D_{i_{t}}$. ) Each subset

$$
\rho \subseteq W
$$

is called $n$-ary relation on $D$. From a practical point of view the finite relations are of interest, i.e. all possible finite subsets of $W$ and therefore in the present piece of writing "relation” will mean ,finite relation" (besides the opposite is explicitly emphasized), no matter it is supposed that the sets $D_{i}, \quad i=1,2, \cdots, m$ could possibly be infinite (for example infinite sets of real numbers).

Let's mark with $R$ the set of all $n$-ary relations formed by $n, n=1,2, \cdots \quad$ (not definitely different) sets (domains) from the family $D$.

Let $\rho \in R$ and let $r^{i}=\left\langle r_{1}^{i}, r_{2}^{i}, \cdots, r_{n}^{i}\right\rangle$ be the $i$-th element of $\rho, r^{i}$ is called the $i$-th record of the relation $\rho$. In this case $i$ is an element index, not an exponent. The component $r_{j}^{i}$ of $r^{i}$ is called value of the $j$-th attribute in the $i$-th record of $\rho$. The values of the $j$-th attributes of all records of $\rho$ make the $j$-th field of $\rho$. From the definition of the term field follows that the elements of $j$-th field can receive values from one sole domain $D_{i_{j}} \in D$.

In the set $R$ of all relations in $D$ in certain circumstances it is possible to define various operations- union, intersection, subtraction, complement, projection, composition, indexing, sorting, etc. Relationships responding to certain conditions are possible between the separate attributes. Thus $R$ together with the introduced operations and relationships between attributes turns into algebra, called relational algebra. Relational algebra is in the base of relational data model. The database management systems, which have the relational model in their bases, are also called relational databases. Basic knowledge in the field of the theory of relational algebras can be obtained in [6].

Each relation $\rho \in R$ could be visually presented like a rectangular table in which the $i$-th row is the $i$-th record, and the $j$-th column is the $j$-th field of $\rho$. This correspondence is one-to-one, i.e. the so built table completely determines the relation presented by it. Due to that reason and to help each user easily understand the main notions of relational algebra, in the most of software manuals for relational database they talk about and operate mostly with the notion table as a synonym of the notion relation in the family of domains [4], never mind that from a practical point of view there could be a greater number of table types and not every table could present concrete relation, i.e. not every type of table could be used in a software for relational database. For example the calendar is a rectangular table, which cannot be identified with the above defined term relation.

\section{Sets of Values and Relation on Them, Which Are Necessary for Creating a Personality Psychological Test}

To create a Personality psychological test, using the help of a computer, it is necessary to define and specify the following sets:

1) The set $Q s t$ is compound of questions or statements, presented for answer or opinion to the tested individual or group of people from the research psychologist. The elements of Qst are the items of the test.

The presenting order of the items has a significant meaning for the psychologist, as the sequence of the discussed items has influence to answers and therefore is important for drawing the final conclusion and test results interpretation. In this relation arises the need of next set:

2) Set

$$
Z_{m}=\{1,2, \cdots, m\}
$$

where $m=|Q s t|$ is the cardinal number of the elements of set $Q s t$. Each element of the set $Z_{m}$ is a unique number of item in the set Qst.

3) There is a bijective mapping $\varphi \subset Q s t \times Z_{m}$ between the sets $Z_{m}$ and $Q s t$, and the author of the psychological test for each $q \in Q s t$ has to define very carefully the image $k=\varphi(q)$ of the element $q$ in the mapping $\varphi$. In this case $k$ is a number of the item $q$ and determines the order of items presentation to the tested individual. As it was above emphasized, determining the number of each item is important for the final conclusion and all that is in the competence of the psychologist author of the test. In addition the number must be in the interval $[1, m]$ of natural numbers. In this sense in a database management system, needed for computer Personality psychological test (Tests generator) development when deleting an item or inserting a new one between two existing items, an automatic items renumbering has to be provided. In this sense the field $Z_{m}$ is quite different from the auto increment field envisaged in a number of database management systems, which serves a primary key. For fields of that type the above mentioned operations division and item insertion are not followed by renumbering, even more it's not allowed to make changes in the primary key value.

4) A finite set Ans of possible answers to the items (see section 1). 
5) A set Ctg of psychological categories (Personality characteristics), which are subject of analysis and evaluation concerning the assessed individual or group of people with the aid of the items from the set Qst and the concrete answer that is chosen. It is not obligatory for each item to be related to given psychological category.

6) We are examining the finite family of subsets of Qst

$$
T=\left\{T_{c} \subseteq Q s t \mid c \in C t g\right\}
$$

with the element $q \in Q s t$ which belongs to the subset $T_{c}$, if and only if the item $q$ has a relation to the category $c \in C t g$ in the correspondent psychological assessment.

Obviously $\bigcup_{c \in C t g} T_{c} \subseteq Q s t$ and if $q \in Q s t$ and

$q \notin \bigcup_{c \in C t g} T_{c}$, then the item $q$ will not effect the entire test and will drop out.

7) Let $A n s=\left\{a_{1}, a_{2}, \cdots, a_{k}\right\}$. For every $a_{i} \in A n s$ we put together the set of numbers $S_{i}$, i.e. we examine the following family of sets of numbers

$$
\left\{S_{i} \mid a_{i} \in A n s, i=1,2, \cdots, k\right\}
$$

We are building the Cartesian product of the sets $S_{i}$, $i=1,2, \cdots, k$ :

$$
\begin{aligned}
S c l & =\prod_{a_{i} \in A n s} S_{i} \\
& =\left\{\left\langle s_{1}, S_{2}, \cdots, s_{k}\right\rangle\left|s_{i} \in S_{i}, i=1,2, \cdots, k, k=\right| \text { Ans } \mid\right\}
\end{aligned}
$$

Each element $s_{i} \in S_{i}, \quad a \in$ Ans from a practical point of view represents an assessment numerical value of the psychological categories from the set Ctg on condition that the assessed person could possibly respond to a random item with an answer or a statement $a \in A n s$. Quite often these assessment values are 1 or 0 . For example, if $|A n s|=2$ and we assume that the assessment value for each positive answer is equal to 1 and for each negative answer is 0 , then we can easily calculate the total number of positive answers.

8) Let's remind that a binary relation $f \subset A \times B$ is called function, if for each $x \in A$ there is no more than one $y \in B$, so that $\langle x, y\rangle \in f$. The fact that $f \subset A \times B$ is a function is presented also like that $f: A \rightarrow B$ and instead of $\langle x, y\rangle \in f$, we write $y=f(x)$.

For each psychological category $c \in C t g$ is defined one function $f_{c}: Q s t \rightarrow S c l$ with the aid of which the psychologist evaluates the assessed person regarding the category $c \in C t g$. Such function is called $a$ scale for the psychological category $c \in C t g$. It's a common practice the name of the scale to be exactly the same as the name of the psychological category. The set of functions $\left\{f_{c} \mid c \in C t g\right\}$, composed by all scales for the examined psychological categories is called a scale of the Personality psychological test.
What is the assessment of the different items, regarding the correspondent answers, i.e. how the functions are defined $f_{c}: Q s t \rightarrow S c l, c \in C t g$ and what is the influence of this to the summary assessment of the test implementation can be estimated after wide psychologycal and statistical investigations [1-3,7].

9) Relation

$$
B n d=\left\{\left\langle c, q, f_{c}(q)\right\rangle \mid c \in C t g, q \in Q s t\right\} \subset C t g \times Q s t \times S c l
$$

where Ctg, Qst and $S c l$ are sets, which are defined correspondingly in subsections 5), 1) and 7), and the relation $f_{c}, c \in C t g$ is a function defined in 8) (scale of psychological category $c$ ). Every record in DBName $B n d$ contains the number of the scores we can take when the person does the corresponding answer $a \in$ Ans according the scale $f_{c}(q)$ for category $c \in C t g$ and item $q \in Q s t$.

10) Let $c \in C t g$ and let

$$
m_{c}=\min \sum_{q \in Q s t} \mu\left(f_{c}(q)\right)
$$

where

$$
\mu\left(\left\langle x_{1}, x_{2}, \cdots, x_{k}\right\rangle\right)=\min \left(x_{1}, x_{2}, \cdots, x_{k}\right)
$$

In other words $m_{c}$ is the minimum assessment value (minimum score), which could possibly be obtained in a random testing, related to the category $c \in C t g$ and depending on the correspondent scale $f_{c}: Q s t \rightarrow S c l$. Analogously we specify the maximum assessment value

$$
M_{c}=\max \sum_{q \in Q s t} v\left(f_{c}(q)\right)
$$

where

$$
v\left(\left\langle x_{1}, x_{2}, \cdots, x_{k}\right\rangle\right)=\max \left(x_{1}, x_{2}, \cdots, x_{k}\right)
$$

Obviously $m_{c}<M_{c}$. We examine the finite sequence of numbers

$$
m_{c}=b_{0}<b_{1}<\cdots<b_{l(c)-1}<b_{l(c)}=M_{c} .
$$

As we denote $l(c)$ we emphasize the fact that the number of the members in the row depends on the category $c \in C t g$. We are separating the interval $\left[m_{c}, M_{c}\right]$ to the subintervals $\left[b_{0}, b_{1}\right],\left(b_{1}, b_{2}\right],\left(b_{2}, b_{3}\right], \cdots$, $\left(b_{l(c)-1}, b_{l(c)}\right]$. Let's mark with $D_{c}$ the set of all intervals of that type $\Delta_{1}=\left[b_{0}, b_{1}\right]$ and $\Delta_{i}=\left(b_{i-1}, b_{i}\right]$, $i=2,3, \cdots, l(c)$, corresponding to the category $c \in C t g$.

The number $l(c)$ and each of the intervals $\Delta_{i}$, $i=1,2, \cdots, l(c)$ should be a result of profound psychological investigations.

Let denote by

$$
D=\bigcup_{c \in C t g} D_{c}
$$

the set of all intervals of numbers, which could be useful for certain personality psychological test. 
11) For each of the intervals $\Delta_{i} \in D_{c}, c \in C t g$ defined in 10), we create text $t_{i}, i=1,2, \cdots, l(c)$, corresponding to the text given by a professional psychologist about the psychological condition of the assessed person regarding the psychological category $c \in C t g$, in case that the total summary (the sum of all concrete evaluation for the corresponding item for the implementing test according to the scale $f_{c}$ ) belongs to the interval $\Delta_{i}$. Thus for each $c \in C t g$ we obtain the sets of texts

$$
T_{c}=\left\{t_{i} \mid i=1,2, \cdots, l(c)\right\}
$$

12) Let $c \in C t g$. By the things we have mentioned above it follows that there exists function

$$
\begin{gathered}
g_{c}: D_{c} \rightarrow T_{c} \\
\text { Let } G_{c}=\left\{\left\langle\Delta_{i}, t_{i}\right\rangle \mid g_{c}\left(\Delta_{i}\right)=t_{i}, i=1,2, \cdots, l(c)\right\}
\end{gathered}
$$

is the corresponding binary relation of this function. The function $g_{c}: D_{c} \rightarrow T_{c}$ is called interpretation of the test, concerning the psychological category $c \in C t g$.

We denote by

$$
T=\bigcup_{c \in C t g} T_{c}
$$

the set of all interpretations, which can be given of the psychological examiner after the implementation of a given personality psychological test.

13) Then we define the relation

$$
N t p=\left\{\left\langle c, \Delta_{i}, g_{c}\left(\Delta_{i}\right)\right\rangle \mid c \in C t g, \Delta_{i} \in D_{c}\right\} \subset C t g \times D \times T
$$

where $D_{c}$ and $D$ are the sets of number intervals, we have already looked at 10), and $g_{c}, c \in C t g$ and $T$ are correspondingly the functions and the set of interpretations, defined in 12).

The sense of each record in Ntp is as follows: For each psychological category $c \in C t g$ when the test is implemented if the assessed person obtain $r$ number of points (obtained assessment value for the category), then we check in which interval of numbers $\Delta_{i} \in D_{c}, c \in C t g$ $r$ is situated and we give the expert interpretation of the test accordingly with the category $c \in C t g$ and the function $g_{c}: D_{c} \rightarrow T_{c}$ defined in 12).

\section{A Software Project for Computer Administering Personality Psychological Tests}

Because of all that is written above, we consider that it is appropriate a computer system for administering personality psychological tests to contain three basic and several contributive, relatively independent modules. How do these separate modules work is shown on a diagram in Figure 1.

1) Module "Generator". This module will create files, containing sets and relations described in section 2 for a concrete personality psychological test. At the moment we do not take an account of the data form, corresponding to the concrete database management system (Oracle Database, dBase, Paradox, FoxPro, MS Access, SQL, etc.). Concrete realization of the module "generator" is described in [8].

2) One or several contributive modules. They serve for additional forming of a concrete computer test - creating starting window, enter a preliminary instruction, description of "demographic field" (if it is necessary) and others. The demographic field is a set of different data, characterizing the examined person as what is his/her sex, age, education, work, settlement, etc.

3) Module "Executor". This is a computer program, which reads the data from files and makes a concrete computer testing. From psychological point of view it is desirable the interface of this program to be as simple as possible. It is enough in the main window to appear the text of the serial item of the set $Q s t$. The order they appear is according to the bijective mapping between the elements of the set $Q s t$ and the set $Z_{m}=\{1,2, \cdots, m\}$, $m=|Q s t|$ in ascending order of the elements in $Z_{m}$, described in subsection 3 ). By request just before the program finishes working appears a window "interpretation", in which are described the conclusions obtained from analyzing the data of the concrete testing according to the relation Ntp described in subsection 13). The data will be recorded in a file, because we will need it for the coming modules of the system for computer administering of psychological tests.

4) Modules for statistic processing. They serve for automated statistic processing of the data, which is obtained when the module "executor" is used many times, testing many persons. We can use universal programs for statistic process, which are often used in practice, such as SPSS, STATISTICA, MatLab, Maple, MS Excel and others. Certainly as we have in mind the specific features of the data, obtained in a result of a computer psychological testing, we consider that it is advisable to create specific software for the concrete statistic processing, which has to read and process the data, obtained when the module "executor" works. This will lead to maximum automation of the psychological examinations.

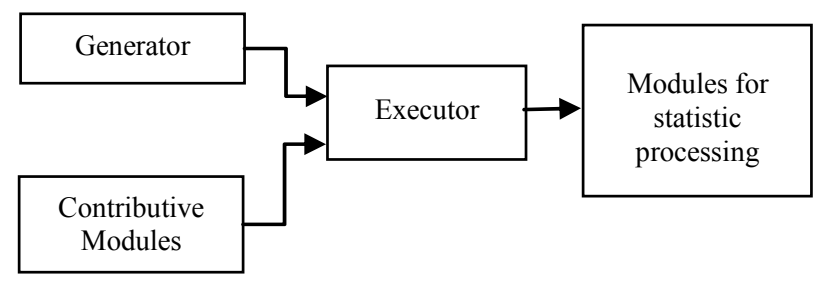

Figure 1. A computer system for administering personality psychological tests. 


\section{Conclusion}

The described mathematical model is a useful task for university studies in databases course. On the other hand, discussed in the work notes will stimulate the psychologists to make wider use of computer methods in their work. This model could be used to develop real software for computer administering of any psychological test and there is full automation of the whole process: test construction, test implementation, result evaluation, storage of the developed database, statistical implementation, analysis and interpretation.

\section{REFERENCES}

[1] E. Sidorenko, "Methods for Mathematical Processing in Psychology," Rech, Sankt Petersburg, 2000.

[2] A. Anastasi and S. Urbina, "Psychological Testing," Prentice-Hall, Englewood Cliffs, 1997.
[3] S. A. Miller, "Developmental Research Methods," 2nd Edition, Prentice-Hall, Englewood Cliffs, 1998.

[4] M. J. Hernandez, "Database Design for Mere Mortals," 2nd Edition, Addison Wesley, Boston, 2003.

[5] E. F. Codd, "A Relational Model of Data for Large Shared Data Banks," Communication of the Association for Computing Machinery, Vol. 13, No. 6, 1970, pp. 377-387. doi: $10.1145 / 362384.362685$

[6] D. Maier, "The Theory of Relational Databases," Computer Science Press, New York, 1983.

[7] A. Nasledov, "Mathematical Methods for Psychological Assessments-Analysis and Data Interpretation," Rech, Sankt Petersburg, 2004.

[8] K. Yordzhev, I. Peneva and B. Kirilieva-Shivarova, "A Relational Model of Personality Psychological Tests," Proceedings of the 3rd International Conference of Faculty of Mathematics \& Natural Science (FMNS2009), Blagoevgrad, 3-7 June 2009, pp. 69-77. 